\title{
一种合成二氢黄酮醇衍生物的简易方法
}

\author{
周宗宝王红陶凯奇叶晓川胡春玲*
}

(湖北中医药大学药学院 中药化学与中药资源省级重点实验室 武汉 430065)

\begin{abstract}
摘要 以 $2^{\prime}$-羊基查尔酮衍生物为原料, 在低温条件下, 以四氢呋喃为溶剂, 在 $\mathrm{H}_{2} \mathrm{O}_{2} /\left(\mathrm{C}_{2} \mathrm{H}_{5}\right)_{2} \mathrm{NH}$ 体系中, 高收率地实现 一系列二氢黄酮醇类衍生物的合成. 考查了反应温度、反应溶剂、碱添加剂的种类和比例以及氧化剂的种类和比例对 该反应的影响，确定了最优反应条件. 此合成方法具有操作简单、条件温和及后处理方便等特点. 此外, 所合成产物的 结构经核磁共振谱表征确证.
\end{abstract}

关键词 2'-差基查尔酮衍生物; 二氢黄酮醇类衍生物; 简易的方法

\section{A Facile Method to Prepare Dihydroflavonol Derivatives}

\author{
Zhou, Zongbao $\quad$ Wang, Hong $\quad$ Tao, Kaiqi $\quad$ Ye, Xiaochuan $\quad$ Hu, Chunling* \\ (Key Laboratory of Chinese Medicine Chemistry and Chinese Herbal Compound of Hubei Province, \\ College of Pharmacy, Hubei University of Traditional Chinese Medicine, Wuhan 430065)
}

\begin{abstract}
A series of dihydroflavonol derivatives were synthesized by the reaction of 2'-hydroxyl chalcone derivatives with $\mathrm{H}_{2} \mathrm{O}_{2} /\left(\mathrm{CH}_{3} \mathrm{CH}_{2}\right)_{2} \mathrm{NH}$ system in anhydrous tetrahydrofuran under low temperature. The influences of reaction temperature, reaction solvents, the kinds and proportion of alkaline additive and oxidants on this reaction were investigated, and the optimal reaction conditions were determined. This method has the advantages of simple operation, mild conditions and convenient post-treatment. Besides, all synthetic products were fully characterized by NMR spectra.
\end{abstract}

Keywords 2'-hydroxyl chalcone derivatives; dihydroflavonol derivatives; facile method

二氢黄酮类化合物为黄酮类 C-2,3 位的双键氢化后 的衍生物, 此类化合物在植物中多数带有羟基或甲氧 基, 当 C-3 位上带有羟基时, 通称为二氢黄酮醇类 (dihydroflavonols). 某些二氢黄酮醇类化合物表现出极 佳的生物活性, 如花旗松素((taxifolin)、落新妇昔 (astilbin)、二氢杨梅素 (dihydromyricetin) 及水飞蓟宾 (silybin, 图 1), 这些二氢黄酮醇类化合物显示出良好的 抗氧化 ${ }^{[1,2]}$ 、抗菌 ${ }^{[3]}$ 、抗肿瘤 ${ }^{[4,5]}$ 等生物活性, 可以作为潜 在的先导化合物进行研究. 二氢黄酮醇类化合物作为一 类微量黄酮类化合物, 在自然界分布相对有限, 提取非 常困难. 因此, 如何有效地合成二氢黄酮醇类化合物就 成为有机和药物化学家迫切关注的问题.

通常制备二氢黄酮醇是采用 Algar-Flynn-Oyamada (AFO)反应 ${ }^{[6 \sim 9]}$. 首先, 在强碱条件下将 $2^{\prime}$-差基查尔酮 衍生物的酚羟基保护起来, 然后制备其相应的环氧化合 物, 最后在酸性条件下脱保护环合生成所需的二氢黄酮
醇. 该方法虽然应用广泛, 但是其要求的反应步骤过于 繁琐, 化合物纯化过程十分麻烦, 反应产率受底物及条 件的影响较大 ${ }^{[10]}$. 有研究报道 ${ }^{[11]}, 2^{\prime}$-羟基查尔酮衍生物 能在铇酸钠的缓冲介质中生成二氢黄酮醇, 但是该方法 所制得二氢黄酮醇产率较低 $(25 \% \sim 28 \%)$, 适用范围以 及 $2^{\prime}$-羟基查尔酮环上取代基的类型受到相当程度的限 制. 也有研究报道 ${ }^{[12,13]}$, 以 $2^{\prime}$-羟基查尔酮衍生物为原料, 以 Dioxane 为溶剂, 在 $\mathrm{H}_{2} \mathrm{O}_{2} /\left(\mathrm{C}_{2} \mathrm{H}_{5}\right)_{2} \mathrm{NH}$ 体系中生成了一 系列二氢黄酮醇，但是同样其产率普遍不高 $(38 \%$ $63 \%)$. 因此, 发展从简单易得的原料出发, 且可以灵活 引入不同取代基的二氢黄酮醇衍生物的合成新方法就 显得尤为重要. 本文以 2 -羟基查尔酮类化合物为原料, 在低温条件下, 以四氢呋喃(THF) 为溶剂, 以 $\mathrm{H}_{2} \mathrm{O}_{2}$ / $\left(\mathrm{C}_{2} \mathrm{H}_{5}\right)_{2} \mathrm{NH}$ 为氧化剂和添加剂, 成功地开发了一种合成 二氢黄酮醇衍生物的简易方法. 合成路线如 Eq. 1.

\footnotetext{
*E-mail: 1054681196@qq.com

Received May 30, 2016; revised June 25, 2016; published online July 15, 2016.

Project supported by the National Natural Science Foundation of China (No. 31370378).

国家自然科学基金(No. 31370378)资助项目.
} 
<smiles>O=C1c2c(O)cc(O)cc2OC(c2ccc(O)c(O)c2)C1O</smiles><smiles>O=C1c2c(O)cc(O)cc2O[C@H](c2ccc3c(c2)OC(c2ccc(O)c(O)c2)C(CO)O3)C1O</smiles><smiles>O=C(C=Cc1ccccc1)c1ccccc1O</smiles><smiles>COONOCCO</smiles><smiles></smiles><smiles>O=C1c2ccccc2OC(c2ccccc2)C1O</smiles><smiles>O=C1/C(=C/c2ccccc2)Oc2ccccc21</smiles>

图 1 具有生物活性的二氢黄酮醇类化合物

Figure 1 Representative examples of biologically active dihydroflavonols<smiles>[R1]C=C1c2cccc(O)c2OC(c2ccc([R])cc2)C1O</smiles>

\section{1 结果与讨论}

\section{1 设计思路}

Oyamada 等 ${ }^{[14]}$ 优化了 $\mathrm{AFO}$ 反应制备二氢黄酮醇, 反应中控制 $\mathrm{pH}=10.5$, 通过 $\mathrm{H}_{2} \mathrm{O}_{2} / \mathrm{NaOH}$ 体系得到了目 标产物. 但是此方法对底物要求十分严格, 所有产物纯 化步骤十分繁琐，因此该方法未受到重视. Tanaka 等 ${ }^{[15]}$ 报道将 2'-差基查尔酮固体均匀分散在水中, 室温下在 $\mathrm{H}_{2} \mathrm{O}_{2} / \mathrm{NaOH}$ 体系中搅拌 $2 \mathrm{~h}$ 能得到高产率的二氢黄酮 醇, 但是我们应用该方法时却未能得到二氢黄酮醇, 而 是只得到相应的氧化产物黄酮醇(flavonol). Cummins 等 [16]报道, 同样的底物在 $\mathrm{H}_{2} \mathrm{O}_{2} / \mathrm{NaOH}$ 体系及甲醇溶液中, 在 $5{ }^{\circ} \mathrm{C}$ 下反应 $3 \mathrm{~h}$ 能得到二氢黄酮醇、黄酮醇和橙酮 (aurone)的混合物. 由此, 我们推测反应温度可能对反 应产物有重要的影响. Patonay 等 ${ }^{[17]}$ 报道, 将 $2^{\prime}$-差基查 尔酮溶解在二氯甲烷和丙酮溶剂中, 在室温及 $\mathrm{H}_{2} \mathrm{O}_{2} / \mathrm{NaOH}$ 体系中搅拌, 合成了部分二氢黄酩醇, 但其 产率较低( $21 \% \sim 24 \%)$. 这一结果再一次给了我们启示: 反应溶剂对反应结果的影响同样不容忽视.

根据 AFO 反应机理可知, 2'-差基查尔酮转化为二氢 黄酮醇涉及到酚着基在碱性条件下活化为酚氧负离子 与双键环氧化这两个过程 ${ }^{[18-20]}$, 而碱添加剂和氧化剂 的种类和比例直接影响这两个反应过程, 因此, 选择合 适的碱添加剂和氧化剂就显得非常重要.

\section{2 反应条件的选择}

综合以上分析, 我们以 $2^{\prime}$-羟基查尔酮为原料, 分别 考查了反应温度、反应溶剂、碱添加剂的种类和比例以 及氧化剂的种类和比例对该反应的影响.

从表 1 中 Entries 1 4 的数据可知, 当温度高于$5{ }^{\circ} \mathrm{C}$ 时, 二氢黄酮醇的产率急剧下降; 当温度低于一 $5{ }^{\circ} \mathrm{C}$ 时, 反应时间延长, 二氢黄酮醇的产率降低; 当温 度保持在 $-5{ }^{\circ} \mathrm{C}$ 时, 二氢黄酮醇的产率最高. 有趣的是 我们将反应完毕后的体系放置室温继续摚拌时, 反应溶 液颜色变深, 生成的二氢黄酮醇会逐渐变为二氢黄酮醇 与黄酮醇的混合物, 并且随着时间的延长, 黄酩醇的量 会逐渐增大, 甚至全部转化为黄酮醇. 这说明反应温度 对该反应影响十分显著, 因此保持反应温度 $-5{ }^{\circ} \mathrm{C}$ 是必 需条件.

从表 1 中 Entries $2,5 \sim 7$ 的数据可知, 溶剂的种类 能够影响到最终的产物类型. 当选用甲醇这种极性质子 性溶剂时，反应最终会生成黄酮醇; 而当选用非质子性 溶剂时 [如丙酮、 $N, N$-二甲基甲酰胺(DMF)和 THF] , 反应 会停留在二氢黄酩醇的阶段, 并且溶剂重蒸处理后二氢 黄酮醇的产率会提高.

从表中的数据可知(表 1 , Entries $2,8 \sim 10$ ), 随着有 机碱的碱性增强, 产率也随之升高, 碱添加剂选用二乙 胺(DEA)时最佳, 其中当碱添加剂比例为 $n$ (2'-差基查尔 酮) : $n(\mathrm{DEA})=1: 3$ 时, 产率最高. 在探究氧化剂的种 类和比例对反应的影响时(表 1, Entries 2,11 14), 选用 了常用的环氧化试剂间氯过氧苯甲酸(M-CPBA), 但 2'羟基查尔酮未发生任何变化, 这可能是因为用过氧酸处 理 $\alpha, \beta$-不饱和酮无法形成环氧化合物, 从而阻碍了反应 进行 ${ }^{[21]}$. 当选择廉价双氧水作氧化剂时, 双氧水可以使 $\alpha, \beta$-不饱和酮顺利氧化成环氧化合物, 且氧化剂双氧水 的比例为 $n$ (2'-羟基查尔酮) $: n\left(\mathrm{H}_{2} \mathrm{O}_{2}\right)=1: 3$ 时, 生成的 二氢黄酮醇的产率最高, 进一步加大 $\mathrm{H}_{2} \mathrm{O}_{2}$ 的量, 二氢黄 
表 1 反应条件的优化

Table 1 Optimization of the reaction conditions ${ }^{a}$<smiles>O=C(/C=C/c1ccccc1)c1ccccc1O</smiles><smiles>CO[Mg]O[Mg]</smiles>

2'- hydroxyl chalcone

1a<smiles>O=C1c2ccccc2OC(c2ccccc2)C1O</smiles>

[O]<smiles>O=c1c(O)c(-c2ccccc2)oc2ccccc12</smiles>

2a

\begin{tabular}{crllcc}
\hline Entry Temp. $/{ }^{\circ} \mathrm{C}$ & Solvent & Alkaline additive & Oxidant $^{b}$ & Yield/\% \\
\hline 1 & -10 & THF & Diethylamine & $\mathrm{H}_{2} \mathrm{O}_{2}$ & 40 \\
2 & -5 & THF & Diethylamine & $\mathrm{H}_{2} \mathrm{O}_{2}$ & 75 \\
3 & 0 & THF & Diethylamine & $\mathrm{H}_{2} \mathrm{O}_{2}$ & 45 \\
4 & 10 & THF & Diethylamine & $\mathrm{H}_{2} \mathrm{O}_{2}$ & 10 \\
5 & -5 & Methanol & Diethylamine & $\mathrm{H}_{2} \mathrm{O}_{2}$ & $0^{c}$ \\
6 & -5 & DMF & Diethylamine & $\mathrm{H}_{2} \mathrm{O}_{2}$ & 35 \\
7 & -5 & Acetone & Diethylamine & $\mathrm{H}_{2} \mathrm{O}_{2}$ & 45 \\
8 & -5 & THF & Diethylamine & $\mathrm{H}_{2} \mathrm{O}_{2}$ & $65^{d}$ \\
9 & -5 & THF & Triethylamine & $\mathrm{H}_{2} \mathrm{O}_{2}$ & 65 \\
10 & -5 & THF & Pyridine & $\mathrm{H}_{2} \mathrm{O}_{2}$ & 67 \\
11 & -5 & THF & Diethylamine & $\mathrm{H}_{2} \mathrm{O}_{2}$ & $61^{e}$ \\
12 & -5 & THF & Diethylamine & $\mathrm{H}_{2} \mathrm{O}_{2}$ & $30^{f}$ \\
13 & -5 & THF & Diethylamine & $\mathrm{M}^{2} \mathrm{CPBA}$ & 0 \\
14 & -5 & THF & Diethylamine & DDO & 66 \\
\hline
\end{tabular}

${ }^{a}$ Unless otherwise specified, all the reactions were carried out using $1.0 \mathrm{mmol}$ of 1a, $300 \mathrm{~mol} \%$ alkaline additive, $300 \mathrm{~mol} \%$ oxidant, $15.0 \mathrm{~mL}$ of solvent; ${ }^{b}$ The mass fraction of $\mathrm{H}_{2} \mathrm{O}_{2}$ is $30 \%$; ${ }^{c}$ The reaction product is flavonol; ${ }^{d} 200$ mol $\%$ alkaline additive; ${ }^{e} 200 \mathrm{~mol} \%$ oxidant; ${ }^{f} 400 \mathrm{~mol} \%$ oxidant.

酮醇的产率急剧下降, 这可能是过量的 $\mathrm{H}_{2} \mathrm{O}_{2}$ 容易将二 氢黄酮醇氧化成了黄酮醇而得不到所需的产物二氢黄 酮醇. 综上所述, 在 THF 溶剂中, 温度保持 $-5{ }^{\circ} \mathrm{C}, n\left(2^{\prime}-\right.$ 羟基查尔酮) $: n$ (DEA) $: n\left(\mathrm{H}_{2} \mathrm{O}_{2}\right)=1: 3: 3$ 时, 是 2'-差弪 基查尔酮转化为二氢黄酮醇的最佳反应条件.

\section{3 底物的拓展}

在上述优化的反应条件下, 我们对该反应底物的普 适性进行了考察. 如表 2 所示, 在该反应体系中, 不同 取代基 2'-羟基查尔酮底物都能适用于该反应体系, 并 且所得二氢黄酮醇的收率也不错. 但底物 2 -羟基查尔 酮两个芳环上( $\mathrm{A}$ 环与 $\mathrm{B}$ 环)是否有取代基、取代基的位 置如何以及取代基的种类, 对反应时间与产率有一定的 影响, 差异如表中所示. 当 2'-差基查尔酮 $\mathrm{A}$ 环取代基相 同(表 2, Entries 1 6, 7 12)，B 环上有给电子基团时， 反应时间延长, 产率降低; 而当 $\mathrm{B}$ 环有吸电子基时, 反 应时间缩短, 产率相应提高(表 2, Entries 3,6,9,12). 这

可能由于反应时间的延长，使生成的二氢黄酮醇更容易 氧化成黄酮醇, 从而降低产率. 此外, 值得注意的是, 当 2'-羟基查尔酮 B 环无取代基时(表 2, Entries 1,7), A 环上存在的给电子基越多，反应时间越短，产率越高. 因此，该反应底物适用范围广泛，为不同取代基的二氢 黄酮醇衍生物的合成提供了简便的新方法.

表 2 底物的拓展 ${ }^{a}$

Table 2 Scope of the substrates

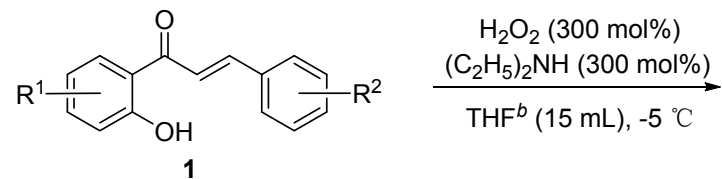<smiles>[R][R]c1ccc(C2Oc3ccc([R])cc3C(=O)C2O)cc1</smiles>

\begin{tabular}{|c|c|c|c|c|c|}
\hline Entry & Product & $\mathrm{R}^{1}$ & $\mathrm{R}^{2}$ & Time $^{c} / \mathrm{h}$ & Yield/\% \\
\hline 1 & $2 a$ & $\mathrm{H}$ & $\mathrm{H}$ & 8 & 75 \\
\hline 2 & $2 b$ & $\mathrm{H}$ & 4'-OMe & 15 & 70 \\
\hline 3 & $2 c$ & $\mathrm{H}$ & 4'-F & 6 & 76 \\
\hline 4 & 2d & $\mathrm{H}$ & $4^{\prime}-\mathrm{CH}\left(\mathrm{CH}_{3}\right)$ & 20 & 71 \\
\hline 5 & $2 \mathrm{e}$ & $\mathrm{H}$ & $3^{\prime}, 4^{\prime}-\mathrm{OMe}$ & 24 & 65 \\
\hline 6 & $2 f$ & $\mathrm{H}$ & $4^{\prime}-\mathrm{Cl}$ & 4 & 84 \\
\hline 7 & $2 g$ & 4-OMe & $\mathrm{H}$ & 4 & 79 \\
\hline 8 & $2 \mathrm{~h}$ & 4-OMe & 4'-OMe & 7 & 75 \\
\hline 9 & $2 i$ & 4-OMe & 4'-F & 3 & 81 \\
\hline 10 & $2 j$ & 4-OMe & $4^{\prime}-\mathrm{CH}\left(\mathrm{CH}_{3}\right)$ & 12 & 67 \\
\hline 11 & $2 k$ & 4-OMe & $3^{\prime}, 4^{\prime}-\mathrm{OMe}$ & 13 & 71 \\
\hline 12 & 2I & 4-OMe & $4^{\prime}-\mathrm{Cl}$ & 2 & 90 \\
\hline
\end{tabular}

${ }^{a}$ Unless otherwise specified, all the reactions were carried out on $1.0 \mathrm{mmol}$ of 1; ${ }^{b}$ Redistilled solvent; ${ }^{c}$ The substrate by TLC proves that the reaction is completed.

\section{2 结论}

以 $2^{\prime}$-羟基查尔酮类化合物为原料, 在 $-5{ }^{\circ} \mathrm{C}$ 条件 下, 以 $\mathrm{THF}$ 为溶剂, 以 $\mathrm{H}_{2} \mathrm{O}_{2} /\left(\mathrm{C}_{2} \mathrm{H}_{5}\right)_{2} \mathrm{NH}$ 为氧化剂和碱添 加剂, 高收率地实现了一系列二氢黄酩醇类衍生物的合 成. 该方法具有操作简单、反应条件温和及后处理方便 等特点，且通过选择不同的反应底物，可以实现 2 '-着基 查尔酮环上不同取代基的灵活引入. 因此，该方法为二 氢黄酩醇衍生物的合成提供了一种新的简便方法，为二 氢黄酮醇类化合物的进一步生物活性研究提供了物质 保障.

\section{3 实验部分}

\section{1 仪器与试剂}

WRS-2 型微机熔点仪 (上海世诺物理光学仪器有限 公司, 温度未经校正); Bruker Advance-400 FT 型核磁共 
振仪(德国 Bruker 公司, 溶剂为 $\mathrm{CDCl}_{3}, \mathrm{TMS}$ 为内标); 紫 外灯检测(UV-8 三用紫外仪); 薄层色谱用硅胶(GF254, 青岛海洋化工厂产品). 其余试剂均为国产化学纯或分 析纯.

\section{2 实验方法}

2'-羟基查尔酮衍生物的合成参考文献[22 24]合 成. 将 2 '-羟基查尔酮衍生物 (1.0 mmol)、二乙胺 $(3.0$ $\mathrm{mmol}$ )和 $10 \mathrm{~mL}$ 无水四氢呋喃 ${ }^{[25]}$ 加入 $50 \mathrm{~mL}$ 茄型反应瓶 中, 搅拌均匀, 在冰盐浴下缓慢滴加 $0.3 \mathrm{~mL} 30 \% \mathrm{H}_{2} \mathrm{O}_{2}$ (3.0 mmol), 滴加完毕后保持温度在 $-5{ }^{\circ} \mathrm{C}$, 继续摚拌 直至反应完全(TLC 检测), 缓慢向反应体系中加入 20 $\mathrm{mL}$ 冷水, 静置, 固体沉淀完全, 过滤, 冷水洗涤, 干燥, 粗产品用石油醚和乙酸乙酯重结晶, 得目标产物 $\mathbf{2 a} \sim$ 21.

2-苯基-3-着基苯并二氢吡喃-4-酮(2a) ${ }^{[12]}$ : 白色针状 结晶, m.p. $186.5 \sim 188.7{ }^{\circ} \mathrm{C} ;{ }^{1} \mathrm{H} \mathrm{NMR}\left(\mathrm{CDCl}_{3}, 400 \mathrm{MHz}\right)$ $\delta: 7.92(\mathrm{~d}, J=7.6 \mathrm{~Hz}, 1 \mathrm{H}), 7.60 \sim 7.40(\mathrm{~m}, 6 \mathrm{H}), 7.16 \sim$ $7.00(\mathrm{~m}, 2 \mathrm{H}), 5.13(\mathrm{~d}, J=12.0 \mathrm{~Hz}, 1 \mathrm{H}), 4.62(\mathrm{~d}, J=12.0$ $\mathrm{Hz}, 1 \mathrm{H}), 3.69(\mathrm{~s}, 1 \mathrm{H}) ;{ }^{13} \mathrm{C}$ NMR $\left(\mathrm{CDCl}_{3}, 100 \mathrm{MHz}\right) \delta$ : $194.23,161.74,136.92,136.36,129.30,128.69,127.57$, 127.35, 122.11, 118.14, 83.91, 73.67.

2-苯基-3-差基-4'-甲氧基苯并二氢吡喃-4-酮 $(\mathbf{2 b})^{[12]}$ : 白色固体, m.p. 166.8 169.0 ${ }^{\circ} \mathrm{C} ;{ }^{1} \mathrm{H} \mathrm{NMR}\left(\mathrm{CDCl}_{3}, 400\right.$ MHz) $\delta: 7.92(\mathrm{~d}, J=7.6 \mathrm{~Hz}, 1 \mathrm{H}), 7.54(\mathrm{dd}, J=8.8,15.6$ $\mathrm{Hz}, 3 \mathrm{H}), 7.10(\mathrm{~d}, J=7.6 \mathrm{~Hz}, 1 \mathrm{H}), 7.02(\mathrm{dd}, J=8.8,15.6$ $\mathrm{Hz}, 3 \mathrm{H}), 5.09$ (d, $J=12.4 \mathrm{~Hz}, 1 \mathrm{H}), 4.64(\mathrm{~d}, J=12.4 \mathrm{~Hz}$, $1 \mathrm{H}), 3.84(\mathrm{~s}, 3 \mathrm{H}), 3.67(\mathrm{~s}, 1 \mathrm{H}) ;{ }^{13} \mathrm{C} \mathrm{NMR}\left(\mathrm{CDCl}_{3}, 100\right.$ MHz) $\delta: 194.39,161.79,160.37,136.90,128.96,127.33$, $122.04,118.15,114.20,83.62,73.60,55.38$.

2-苯基-3-羟基-4'-氟苯并二氢吡喃-4-酮 (2c) ${ }^{[26]}$ : 白 色固体, m.p. $164.0 \sim 166.7{ }^{\circ} \mathrm{C} ;{ }^{1} \mathrm{H}$ NMR $\left(\mathrm{CDCl}_{3}, 400\right.$ MHz) $\delta$ : $7.92(\mathrm{~d}, J=7.6 \mathrm{~Hz}, 1 \mathrm{H}), 7.57(\mathrm{~d}, J=8.4 \mathrm{~Hz}, 2 \mathrm{H})$, $7.55(\mathrm{~s}, 1 \mathrm{H}), 7.15(\mathrm{~s}, 2 \mathrm{H}), 7.10(\mathrm{~d}, J=7.6 \mathrm{~Hz}, 1 \mathrm{H}), 7.04(\mathrm{~d}$, $J=8.4 \mathrm{~Hz}, 1 \mathrm{H}), 5.12(\mathrm{~d}, J=12.4 \mathrm{~Hz}, 1 \mathrm{H}), 4.57(\mathrm{~d}, J=12.4$ $\mathrm{Hz}, 1 \mathrm{H}), 3.74(\mathrm{~s}, 1 \mathrm{H}) ;{ }^{13} \mathrm{C}$ NMR $\left(\mathrm{CDCl}_{3}, 100 \mathrm{MHz}\right) \delta$ : 194.06, 164.43, 161.96, 161.58, 137.03, 132.29, 129.34, 127.39, 122.26, 118.49, 118.11, 115.57, 83.13, 73.68.

$4^{\prime}$-异丙基-2-苯基-3-羟基苯并二氢吡喃-4-酮 (2d $)^{[26]}$ : 白色固体, m.p. 159.7 163.2 ${ }^{\circ} \mathrm{C} ;{ }^{1} \mathrm{H}$ NMR $\left(\mathrm{CDCl}_{3}, 400\right.$ MHz) $\delta: 7.93(\mathrm{~d}, J=7.6 \mathrm{~Hz}, 1 \mathrm{H}), 7.53(\mathrm{dd}, J=7.6,8.4 \mathrm{~Hz}$, $3 \mathrm{H}), 7.32$ (s, 2H), 7.10 (d, $J=8.4 \mathrm{~Hz}, 1 \mathrm{H}), 7.04$ (d, $J=8.4$ $\mathrm{Hz}, 1 \mathrm{H}), 5.12(\mathrm{~d}, J=12.4 \mathrm{~Hz}, 1 \mathrm{H}), 4.67(\mathrm{~d}, J=12.4 \mathrm{~Hz}$, $1 \mathrm{H}), 3.68$ (s, 1H), 2.96 (p, $J=6.8 \mathrm{~Hz}, 1 \mathrm{H}), 1.28(\mathrm{~s}, 6 \mathrm{H})$; ${ }^{13} \mathrm{C}$ NMR $\left(\mathrm{CDCl}_{3}, 100 \mathrm{MHz}\right) \delta: 194.37,161.81,150.16$, $136.92,133.58,127.59,126.91,122.05,118.16,83.85$,
73.56, 34.03, 23.92.

2-苯基-3-羟基-3',4'-二甲氧基苯并二氢吡喃-4-酮 $(2 \mathrm{e})^{[26]}$ : 白色固体, m.p. $159.7 \sim 60.5{ }^{\circ} \mathrm{C} ;{ }^{1} \mathrm{H}$ NMR $\left(\mathrm{CDCl}_{3}, 400 \mathrm{MHz}\right) \delta: 7.92(\mathrm{~d}, J=7.6 \mathrm{~Hz}, 1 \mathrm{H}), 7.55(\mathrm{~d}, J=$ $7.6 \mathrm{~Hz}, 1 \mathrm{H}), 7.18 \sim 7.00(\mathrm{~m}, 4 \mathrm{H}), 6.95(\mathrm{~s}, 1 \mathrm{H}), 5.08(\mathrm{~d}, J=$ $12.4 \mathrm{~Hz}, 1 \mathrm{H}), 4.65(\mathrm{~d}, J=12.4 \mathrm{~Hz}, 1 \mathrm{H}), 3.92(\mathrm{~d}, J=5.8$ $\mathrm{Hz}, 6 \mathrm{H}), 3.71(\mathrm{~s}, 1 \mathrm{H}) ;{ }^{13} \mathrm{C} \mathrm{NMR}\left(\mathrm{CDCl}_{3}, 100 \mathrm{MHz}\right) \delta$ : $194.28,161.70,149.89,149.21,136.92,128.69,127.35$, $122.10,120.47,118.15,111.13,110.34,83.91,73.60$, 55.96 .

2-苯基-3-羟基-4'-氯苯并二氢吡喃-4-酮 $(2 \mathbf{f})^{[26]}$ : 白 色固体, m.p. 172.4 173.7 ${ }^{\circ} \mathrm{C} ;{ }^{1} \mathrm{H}$ NMR $\left(\mathrm{CDCl}_{3}, 400\right.$ MHz) $\delta$ : $7.92(\mathrm{~d}, J=7.6 \mathrm{~Hz}, 1 \mathrm{H}), 7.52(\mathrm{~d}, J=8.4 \mathrm{~Hz}, 2 \mathrm{H})$, $7.43(\mathrm{~d}, J=8.4 \mathrm{~Hz}, 2 \mathrm{H}), 7.12(\mathrm{~d}, J=7.6 \mathrm{~Hz}, 1 \mathrm{H}), 7.05$ (s, $1 \mathrm{H}), 5.11(\mathrm{~d}, J=12.4 \mathrm{~Hz}, 1 \mathrm{H}), 4.55(\mathrm{~d}, J=12.4 \mathrm{~Hz}, 1 \mathrm{H})$, $3.72(\mathrm{~s}, 1 \mathrm{H}) ;{ }^{13} \mathrm{C}$ NMR $\left(\mathrm{CDCl}_{3}, 100 \mathrm{MHz}\right) \delta: 193.92$, $161.53,137.05,134.92,128.88,127.40,122.32,118.47$, 118.12, 83.07, 73.64 .

2-苯基-3-羟基-4-甲氧基苯并二氢吡喃-4-酮 $(2 \mathrm{~g})^{[12]}$ : 白色固体, m.p. $139.9 \sim 142.1{ }^{\circ} \mathrm{C} ;{ }^{1} \mathrm{H}$ NMR $\left(\mathrm{CDCl}_{3}, 400\right.$ MHz) $\delta$ : $7.92(\mathrm{~s}, 1 \mathrm{H}), 7.60 \sim 7.40(\mathrm{~m}, 6 \mathrm{H}), 7.16 \sim 7.00(\mathrm{~m}$, $2 \mathrm{H}), 5.13(\mathrm{~d}, J=12.0 \mathrm{~Hz}, 1 \mathrm{H}), 4.62(\mathrm{~d}, J=12.0 \mathrm{~Hz}, 1 \mathrm{H})$, $3.69(\mathrm{~s}, 1 \mathrm{H}) ;{ }^{13} \mathrm{C}$ NMR $\left(\mathrm{CDCl}_{3}, 100 \mathrm{MHz}\right) \delta: 192.77$, $166.84,163.82,135.92,136.36,128.57 .30,127.54,110.64$, $100.67,84.37,73.05,55.40$.

2-苯基-3-羟基-4,4'-二甲氧基苯并二氢吡喃-4-酮 (2h) ${ }^{[12]}$ : 白色固体, m.p. $134.9 \sim 136.4{ }^{\circ} \mathrm{C} ;{ }^{1} \mathrm{H}$ NMR $\left(\mathrm{CDCl}_{3}, 400 \mathrm{MHz}\right) \delta: 7.84(\mathrm{~d}, J=8.8 \mathrm{~Hz}, 1 \mathrm{H}), 7.50$ (d, $J=$ $8.4 \mathrm{~Hz}, 2 \mathrm{H}), 6.99$ (d, $J=8.4 \mathrm{~Hz}, 2 \mathrm{H}), 6.65(\mathrm{~d}, J=8.8 \mathrm{~Hz}$, $1 \mathrm{H}), 6.47(\mathrm{~s}, 1 \mathrm{H}), 5.05(\mathrm{~d}, J=12.4 \mathrm{~Hz}, 1 \mathrm{H}), 4.56(\mathrm{~d}, J=$ $12.4 \mathrm{~Hz}, 1 \mathrm{H}), 3.83$ (s, 6H), 3.73 (s, 1H); ${ }^{13} \mathrm{C} \mathrm{NMR}\left(\mathrm{CDCl}_{3}\right.$, $100 \mathrm{MHz}) \delta$ : $192.68,166.83,163.89,160.34,128.95$, $114.19,112.13,110.90,100.98,83.82,73.12,55.76,55.37$.

2-苯基-3-羟基-4-甲氧基-4'-氟苯并二氢吡喃-4-酮 (2i) ${ }^{[27]}$ : 白色固体, m.p. $129.3 \sim 129.7{ }^{\circ} \mathrm{C} ;{ }^{1} \mathrm{H}$ NMR $\left(\mathrm{CDCl}_{3}, 400 \mathrm{MHz}\right) \delta: 7.85(\mathrm{~d}, J=8.8 \mathrm{~Hz}, 1 \mathrm{H}), 7.56(\mathrm{~d}, J=$ $8.4 \mathrm{~Hz}, 2 \mathrm{H}), 7.15$ (d, $J=8.4 \mathrm{~Hz}, 2 \mathrm{H}), 6.67(\mathrm{~d}, J=8.8 \mathrm{~Hz}$, $1 \mathrm{H}), 6.49(\mathrm{~s}, 1 \mathrm{H}), 5.09(\mathrm{~d}, J=12.4 \mathrm{~Hz}, 1 \mathrm{H}), 4.51(\mathrm{~d}, J=$ $12.4 \mathrm{~Hz}, 1 \mathrm{H}), 3.85$ (s, 3H), $3.76(\mathrm{~s}, 1 \mathrm{H}) ;{ }^{13} \mathrm{C} \mathrm{NMR}\left(\mathrm{CDCl}_{3}\right.$, $100 \mathrm{MHz}) \delta$ : 192.30, 166.93, 163.69, 161.95, 132.39, $129.39,129.03,115.56,112.04,111.07,101.00,83.33$, 73.20, 55.79 .

4'-异丙基-2-苯基-3-羟基-4-甲氧基苯并二氢吡喃-4酮(2J) ${ }^{[27]}$ : 白色固体, m.p. $150.8 \sim 151.4{ }^{\circ} \mathrm{C} ;{ }^{1} \mathrm{H}$ NMR $\left(\mathrm{CDCl}_{3}, 400 \mathrm{MHz}\right) \delta: 7.85(\mathrm{~d}, J=8.8 \mathrm{~Hz}, 1 \mathrm{H}), 7.50(\mathrm{~d}, J=$ 
$8.0 \mathrm{~Hz}, 2 \mathrm{H}), 7.33$ (d, $J=8.0 \mathrm{~Hz}, 2 \mathrm{H}), 6.66(\mathrm{~d}, J=8.8 \mathrm{~Hz}$, $1 \mathrm{H}), 6.48$ (s, 1H), 5.09 (d, $J=12.4 \mathrm{~Hz}, 1 \mathrm{H}), 4.59$ (d, $J=$ $12.4 \mathrm{~Hz}, 1 \mathrm{H}), 3.82$ (s, 3H), 3.73 (s, 1H), 2.95 (p, $J=6.8$ $\mathrm{Hz}, 1 \mathrm{H}), 1.27$ (d, $J=6.8 \mathrm{~Hz}, 6 \mathrm{H}) ;{ }^{13} \mathrm{C} \mathrm{NMR}\left(\mathrm{CDCl}_{3}, 100\right.$ $\mathrm{MHz}) \delta: 192.66,166.85,163.91,150.11,133.70,128.98$, $127.58,126.90,112.13,110.90,101.00,84.06,73.07$, $55.75,34.03,23.96$.

2-苯基-3-差基-4,3',4'-三甲氧基苯并二氢吡喃-4-酮 $(2 k)^{[27]}$ : 白色固体, m.p. $147.2 \sim 148.1{ }^{\circ} \mathrm{C} ;{ }^{1} \mathrm{H} \mathrm{NMR}$ $\left(\mathrm{CDCl}_{3}, 400 \mathrm{MHz}\right) \delta: 7.85(\mathrm{~d}, J=8.8 \mathrm{~Hz}, 1 \mathrm{H}), 7.20 \sim 7.04$ $(\mathrm{m}, 2 \mathrm{H}), 6.98 \sim 6.92(\mathrm{~m}, 1 \mathrm{H}), 6.66(\mathrm{~d}, J=8.8 \mathrm{~Hz}, 1 \mathrm{H})$, $6.50(\mathrm{~s}, 1 \mathrm{H}), 5.06(\mathrm{~d}, J=12.4 \mathrm{~Hz}, 1 \mathrm{H}), 4.58(\mathrm{~d}, J=12.4$ Hz, 1H), 3.93 (d, J=10.0 Hz, 6H), 3.84 (s, 3H), 3.74 (s, 1H). ${ }^{13} \mathrm{C} \mathrm{NMR}\left(\mathrm{CDCl}_{3}, 100 \mathrm{MHz}\right) \delta: 192.56,166.86$, $163.82,149.87,149.21,128.98,120.45,112.10,111.14$, 110.30, 101.01, 84.12, 73.10, 55.99.

2-苯基-3-羟基-4-甲氧基-4'-氯苯并二氢吡喃-4-酮 $(2 \mathrm{l})^{[27]}$ : 白色固体, m.p. $110.9 \sim 112.5{ }^{\circ} \mathrm{C} ;{ }^{1} \mathrm{H} \mathrm{NMR}$ $\left(\mathrm{CDCl}_{3}, 400 \mathrm{MHz}\right) \delta: 7.92(\mathrm{~d}, J=7.6 \mathrm{~Hz}, 1 \mathrm{H}), 7.52$ (d, $J=$ $8.4 \mathrm{~Hz}, 2 \mathrm{H}), 7.43$ (d, $J=8.4 \mathrm{~Hz}, 2 \mathrm{H}), 7.12$ (d, $J=7.6 \mathrm{~Hz}$, 1H), 7.05 (s, 1H), $5.11(\mathrm{~d}, J=12.4 \mathrm{~Hz}, 1 \mathrm{H}), 4.55$ (d, $J=$ $12.4 \mathrm{~Hz}, 1 \mathrm{H}), 3.72(\mathrm{~s}, 1 \mathrm{H}) ;{ }^{13} \mathrm{C} \mathrm{NMR}\left(\mathrm{CDCl}_{3}, 100 \mathrm{MHz}\right) \delta$ : $193.92,161.53,137.05,134.92,128.88,127.40,122.32$, $118.47,118.12,83.07,73.64$.

\section{辅助材料(Supporting Information) 化合物 $\mathbf{2 a} \sim 21$ 的} NMR 谱图. 这些材料可以免费从本刊网站 (http:// sioc-journal.cn/)上下载.

\section{References}

[1] Hiroyuki, H.; Yasutomo, M.; Sachiko, S.; Hitoshi, M.; Yukiyoshi, T.; Kenji, M.; Osamu, T.; Chou, W.-H. Biosci. Biotechnol. Biochem. 1996, 60, 945 .

[2] Sugihara, N.; Arakawa, T.; Ohnishi, M.; Furuno, K. Free Radical Biol. Med. 1999, 27, 1313

[3] Matsumoto, T.; Tahara, S. Nippon Nogeikagaku Kaishi. 2001, 75, 659.
[4] Devi, M. A.; Das, N. P. Cancer Lett. 1993, 69, 191.

[5] Kandaswami, C.; Perkins, E.; Drzewiecki, G.; Soloniuk, D. S.; Middleton, E. J. Anti-Cancer Drugs. 1992, 3, 525.

[6] Mecocci, P.; Polidori, M. C.; Praticó, D. Biochim. Biophys. Acta. 2012, 18, 631 .

[7] Sato, M.; Murakami, K.; Uno, M.; Nakagawa, Y.; Katayama, S.; Akagi, K. I.; Masuda, Y.; Takegoshi, K.; Irie, K. J. Biol. Chem. 2013, 288, 23213.

[8] Shi, L.; Feng, X. E.; Cui, J. R.; Fang, L. H.; Du, G. H.; Li, Q. S Bioorg. Med. Chem. Lett. 2010, $20,5466$.

[9] Pandurangan, N.; Bose, C.; Banerji, A. Bioorg. Med. Chem. Lett. 2011, 21, 5328.

[10] Mei, Q. G.; Yuan, W. C.; Wang, C. Chin. J. Org. Chem. 2015, 35, 70 (in Chinese). (梅青刚，袁伟成，王淳，有机化学，2015, 35, 70.)

[11] Brown, N. B.; Macbride, J. A. H. J. Chem. Soc. 1964, 3822.

[12] Shabnam, S.; Makrandl, J. K.; Grover, S. K. Synthesis 1985, 11, 110 .

[13] Loser, R.; Chlupacova, M.; Marecek, A.; Opletalova, V.; Gutschow, M. Helv. Chim. Acta. 2004, 87, 2597.

[14] Oyamada, T.; Baba, H.; Koyama, T. Bull. Yamagata Univ., Eng. 1967, 9, 351

[15] Tanaka, K.; Sugino, T. Green Chem. 2001, 3, 133.

[16] Cummins, B.; Donnelly, D. M. X.; Eades, J. F.; Fletcher, H.; Cinné ide, F. O.; Philbin, E. M.; Swirski, J.; Wheeler, T. S.; Wilson, R. K. Tetrahedron 1963, 19, 499

[17] Patonay, T.; Tóth, G.; Adam, W. Tetrahedron Lett. 1993, 34, 5055.

[18] Algar, J.; Flynn, J. P. Proc. R. Ir. Acad. 1934, B42, 1.

[19] Oyamada, T. J. Chem. Soc. Jpn. 1934, 55, 1256.

[20] Geissman, T. A.; Fukushima, D. K. J. Am. Chem. Soc. 1948, 70, 1686.

[21] Hart, H.; Verma, M.; Wang, I. J. Org. Chem. 1973, 38, 3418.

[22] Tanaka, H.; Stohlmeyer, M. M.; Wandless, T. J.; Taylor, L. P. Tetrahedron Lett. 2000, 41, 9735.

[23] Miao, C. X.; Hong, Y. Y.; Deng, Y. H. Chem. Reag. 2008, 30, 63 (in Chinese).

(苗彩霞, 洪锯裕, 邓优华, 化学试剂, 2008, 30, 63.)

[24] Miao, T. G.; Wang, Q. A.; Fang, W. Q. Chin. J. Org. Chem. 2006, 26, 685 (in Chinese).

(廖头根, 汪秋安, 方伟琴, 有机化学, 2006, 26, 685.)

[25] Cabrera, M.; Simoens, M.; Falchi, G.; Lavaggi, L.; Piroo, E.; Eduardo, E.-E. Bioorg. Med. Chem. 2007, 15, 3356

[26] Mari, J.-C.; Xiong, Y.; Geanna, K. M.; Adam, R. Y.; Tohru T.; Nina, B.; Scott, E. S.; John, A. P. J. Org. Chem. 2010, 75, 4584.

[27] Su, S.; Acquilano, D. E.; Arumugasamy, J.; Beeler, A. B.; Eastwood, E. L.; Giguere, J. R.; Lan, P.; Lei, X.; Min, G. K.; Yeager, A. R.; Zhou, Y.; Panek, J. S.; Snyder, J. K.; Schaus, S. E.; Porco, J. A. Jr. Org. Lett. 2005, 7, 2751. 